\title{
Fixed point theory for the cyclic weaker Meir- Keeler function in complete metric spaces
}

\author{
Chi-Ming Chen
}

Correspondence: ming@mail.nhcue. edu.tw

Department of Applied

Mathematics, National Hsinchu University of Education, No. 521,

Nanda Rd., Hsinchu City 300,

Taiwan

\section{Abstract}

In this article, we introduce the notions of cyclic weaker $\phi \circ \phi$-contractions and cyclic weaker $(\phi, \phi)$-contractions in complete metric spaces and prove two theorems which assure the existence and uniqueness of a fixed point for these two types of contractions. Our results generalize or improve many recent fixed point theorems in the literature.

MSC: 47H10; 54C60; 54H25; 55M2O.

Keywords: fixed point theory, weaker Meir-Keeler function, cyclic weaker $\phi \circ \phi$-contraction, cyclic weaker $(\phi, \phi)$-contraction

\section{Introduction and preliminaries}

Throughout this article, by $\mathbb{R}^{+}, \mathbb{R}$ we denote the sets of all nonnegative real numbers and all real numbers, respectively, while $\mathbb{N}$ is the set of all natural numbers. Let $(X, d)$ be a metric space, $D$ be a subset of $X$ and $f: D \rightarrow X$ be a map. We say $f$ is contractive if there exists $\alpha \in[0,1)$ such that for all $x, y \in D$,

$$
d(f x, f y) \leq \alpha \cdot d(x, y) .
$$

The well-known Banach's fixed point theorem asserts that if $D=X, f$ is contractive and $(X, d)$ is complete, then $f$ has a unique fixed point in $X$. It is well known that the Banach contraction principle [1] is a very useful and classical tool in nonlinear analysis. In 1969, Boyd and Wong [2] introduced the notion of $\Phi$-contraction. A mapping $f$ : $X \rightarrow X$ on a metric space is called $\Phi$-contraction if there exists an upper semi-continuous function $\Phi:[0, \infty) \rightarrow[0, \infty)$ such that

$$
d(f x, f y) \leq \Phi(d(x, y)) \text { for all } \quad x, y \in X .
$$

Generalization of the above Banach contraction principle has been a heavily investigated branch research. (see, e.g., [3,4]). In 2003, Kirk et al. [5] introduced the following notion of cyclic representation.

Definition 1 [5] Let $X$ be a nonempty set, $m \in \mathbb{N}$ and $f: X \rightarrow X$ an operator. Then $X=\cup_{i=1}^{m} A_{i}$ is called a cyclic representation of $X$ with respect to $f$ if

(1) $A_{i}, i=1,2, \ldots, m$ are nonempty subsets of $X$;

(2) $f\left(A_{1}\right) \subset A_{2}, f\left(A_{2}\right) \subset A_{3}, \ldots, f\left(A_{m-1}\right) \subset A_{m}, f\left(A_{m}\right) \subset A_{1}$.

(c) 2012 Chen; licensee Springer. This is an Open Access article distributed under the terms of the Creative Commons Attribution License (http://creativecommons.org/licenses/by/2.0), which permits unrestricted use, distribution, and reproduction in any medium, provided the original work is properly cited. 
Kirk et al. [5] also proved the below theorem.

Theorem 1 [5]Let $(X, d)$ be a complete metric space, $m \in \mathbb{N}, A_{1}, A_{2}, \ldots, A_{m}$, closed nonempty subsets of $X$ and $X=\cup_{i=1}^{m} A_{i}$. Suppose that $f$ satisfies the following condition.

$$
d(f x, f y) \leq \psi(d(x, y)), \quad \text { for all } \quad x \in A_{i}, \quad y \in A_{i+1}, \quad i \in\{1,2, \ldots, m\},
$$

where $\psi:[0, \infty) \rightarrow[0, \infty)$ is upper semi-continuous from the right and $0 \leq \psi(t)<t$ for $t>0$. Then, f has a fixed point $z \in \cap_{i=1}^{n} A_{i}$.

Recently, the fixed theorems for an operator $f: X \rightarrow X$ that defined on a metric space $X$ with a cyclic representation of $X$ with respect to $f$ had appeared in the literature. (see, e.g., [6-10]). In 2010, Păcurar and Rus [7] introduced the following notion of cyclic weaker $\phi$-contraction.

Definition 2 [7] Let $(X, d)$ be a metric space, $m \in \mathbb{N}, A_{1}, A_{2}, \ldots, A_{m}$ closed nonempty subsets of $X$ and $X=\cup_{i=1}^{m} A_{i}$. An operator $f: X \rightarrow X$ is called a cyclic weaker $\phi$-contraction if

(1) $X=\cup_{i=1}^{m} A_{i}$ is a cyclic representation of $X$ with respect to $f$;

(2) there exists a continuous, non-decreasing function $\phi:[0, \infty) \rightarrow[0, \infty)$ with $\phi(t)>0$ for $t \in(0, \infty)$ and $\phi(0)=0$ such that

$$
d(f x, f y) \leq d(x, y)-\varphi(d(x, y)),
$$

for any $x \in A_{i}, y \in A_{i+1}, i=1,2, \ldots, m$ where $A_{m+1}=A_{1}$.

And, Păcurar and Rus [7] proved the below theorem.

Theorem 2 [7]Let $(X, d)$ be a complete metric space, $m \in \mathbb{N}, A_{1}, A_{2}, \ldots, A_{m}$ closed nonempty subsets of $X$ and $X=\cup_{i=1}^{m} A_{i}$. Suppose that $f$ is a cyclic weaker $\phi$-contraction. Then, $f$ has a fixed point $z \in \cap_{i=1}^{n} A_{i}$.

In this article, we also recall the notion of Meir-Keeler function (see [11]). A function $\phi:[0, \infty) \rightarrow[0, \infty)$ is said to be a Meir-Keeler function if for each $\eta>0$, there exists $\delta$ $>0$ such that for $t \in[0, \infty)$ with $\eta \leq t<\eta+\delta$, we have $\phi(t)<\eta$. We now introduce the notion of weaker Meir-Keeler function $\phi:[0, \infty) \rightarrow[0, \infty)$, as follows:

Definition 3 We call $\phi:[0, \infty) \rightarrow[0, \infty)$ a weaker Meir-Keeler function if for each $\eta>0$, there exists $\delta>0$ such that for $t \in[0, \infty)$ with $\eta \leq t<\eta+\delta$, there exists $n_{0} \in \mathbb{N}$ such that $\phi^{n_{0}}(t)<\eta$.

\section{Fixed point theory for the cyclic weaker $\phi \circ \phi$-contractions}

The main purpose of this section is to present a generalization of Theorem 1 . In the section, we let $\phi:[0, \infty) \rightarrow[0, \infty)$ be a weaker Meir-Keeler function satisfying the following conditions:

$\left(\phi_{1}\right) \phi(t)>0$ for $t>0$ and $\phi(0)=0$;

$\left(\phi_{2}\right)$ for all $t \in(0, \infty),\left\{\phi^{n}(t)\right\}_{n \in \mathbb{N}}$ is decreasing;

$\left(\phi_{3}\right)$ for $t_{n} \in[0, \infty)$, we have that
(a) if $\lim _{n \rightarrow \infty} t_{n}=\gamma>0$, then $\lim _{n \rightarrow \infty} \phi\left(t_{n}\right)<\gamma$, and
(b) if $\lim _{n \rightarrow \infty} t_{n}=0$, then $\lim _{n \rightarrow \infty} \phi\left(t_{n}\right)=0$.

And, let $\phi:[0, \infty) \rightarrow[0, \infty)$ be a non-decreasing and continuous function satisfying 
$\left(\phi_{1}\right) \phi(t)>0$ for $t>0$ and $\phi(0)=0$;

$\left(\phi_{2}\right) \phi$ is subadditive, that is, for every $\mu_{1}, \mu_{2} \in[0, \infty), \phi\left(\mu_{1}+\mu_{2}\right) \leq \phi\left(\mu_{1}\right)+\phi\left(\mu_{2}\right)$;

$\left(\phi_{3}\right)$ for all $t \in(0, \infty), \lim _{n \rightarrow \infty} t_{n}=0$ if and only if $\lim _{n \rightarrow \infty} \phi\left(t_{n}\right)=0$.

We state the notion of cyclic weaker $\phi \circ \phi$-contraction, as follows:

Definition 4 Let $(X, d)$ be a metric space, $m \in \mathbb{N}, A_{1}, A_{2}, \ldots, A_{m}$ nonempty subsets of $X$ and $X=\cup_{i=1}^{m} A_{i}$. An operator $f: X \rightarrow X$ is called a cyclic weaker $\phi \circ \phi$-contraction if

(i) $X=\cup_{i=1}^{m} A_{i}$ is a cyclic representation of $X$ with respect to $f$;

(ii) for any $x \in A_{i}, y \in A_{i+1}, i=1,2, \ldots, m$,

$$
\varphi(d(f x, f y)) \leq \phi(\varphi(d(x, y)))
$$

where $A_{m+1}=A_{1}$.

Theorem 3 Let $(X, d)$ be a complete metric space, $m \in \mathbb{N}, A_{1}, A_{2}, \ldots, A_{m}$ nonempty subsets of $X$ and $X=\cup_{i=1}^{m} A_{i}$. Let $f: X \rightarrow X$ be a cyclic weaker $\phi \circ \phi$-contraction. Then, $f$ has a unique fixed point $z \in \cap_{i=1}^{m} A_{i}$.

Proof. Given $x_{0}$ and let $x_{n+1}=f x_{n}=f^{n+1} x_{0}$, for $n \in \mathbb{N} \cup\{0\}$. If there exists $n_{0} \in \mathbb{N} \cup\{0\}$ such that $x_{n_{0}+1}=x_{n_{0}}$, then we finished the proof. Suppose that $x_{n+1} \neq x_{n}$ for any $n \in \mathbb{N}$ $\cup\{0\}$. Notice that, for any $n>0$, there exists $i_{n} \in\{1,2, \ldots, m\}$ such that $x_{n-1} \in A_{i_{n}}$ and $x_{n} \in A_{i_{n}+1}$. Since $f: X \rightarrow X$ is a cyclic weaker $\phi \circ \phi$-contraction, we have that for all $n \in \mathbb{N}$

$$
\begin{aligned}
\varphi\left(d\left(x_{n}, x_{n+1}\right)\right) & =\varphi\left(d\left(f x_{n-1}, f x_{n}\right)\right) \\
& \leq \phi\left(\varphi\left(d\left(x_{n-1}, x_{n}\right)\right)\right),
\end{aligned}
$$

and so

$$
\begin{aligned}
\varphi\left(d\left(x_{n}, x_{n+1}\right)\right) & \leq \phi\left(\varphi\left(d\left(x_{n-1}, x_{n}\right)\right)\right) \\
& \leq \phi\left(\phi\left(\varphi\left(d\left(x_{n-2}, x_{n-1}\right)\right)\right)=\phi^{2}\left(\varphi\left(\left(d\left(x_{n-2}, x_{n-1}\right)\right)\right)\right.\right. \\
& \leq \ldots \ldots \\
& \leq \phi^{n}\left(\varphi\left(d\left(x_{0}, x_{1}\right)\right)\right) .
\end{aligned}
$$

Since $\left\{\phi^{n}\left(\phi\left(d\left(x_{0}, x 1\right)\right)\right)\right\}_{n \in \mathbb{N}}$ is decreasing, it must converge to some $\eta \geq 0$. We claim that $\eta=0$. On the contrary, assume that $\eta>0$. Then by the definition of weaker Meir-Keeler function $\phi$, there exists $\delta>0$ such that for $x_{0}, x_{1} \in X$ with $\eta \leq \phi\left(d\left(x_{0}\right.\right.$, $\left.\left.x_{1}\right)\right)<\delta+\eta$, there exists $n_{0} \in \mathbb{N}$ such that $\phi^{n_{0}}\left(\varphi\left(d\left(x_{0}, x_{1}\right)\right)\right)<\eta$. Since $\lim _{n \rightarrow \infty} \phi^{n}(\phi(d$ $\left.\left.\left(x_{0}, x_{1}\right)\right)\right)=\eta$, there exists $p_{0} \in \mathbb{N}$ such that $\eta \leq \phi^{p}\left(\phi\left(d\left(x_{0}, x_{1}\right)\right)<\delta+\eta\right.$, for all $p \geq p_{0}$. Thus, we conclude that $\phi^{p_{0}+n_{0}}\left(\varphi\left(d\left(x_{0}, x_{1}\right)\right)\right)<\eta$. So we get a contradiction. Therefore $\lim _{n \rightarrow \infty} \phi^{n}\left(\phi\left(d\left(x_{0}, x_{1}\right)\right)\right)=0$, that is,

$$
\lim _{n \rightarrow \infty} \varphi\left(d\left(x_{n}, x_{n+1}\right)\right)=0 .
$$

Next, we claim that $\left\{x_{n}\right\}$ is a Cauchy sequence. We claim that the following result holds:

Claim: for each $\varepsilon>0$, there is $n_{0}(\varepsilon) \in \mathbb{N}$ such that for all $p, q \geq n_{0}(\varepsilon)$,

$$
\varphi\left(d\left(x_{p}, x_{q}\right)\right)<\varepsilon,
$$


We shall prove (*) by contradiction. Suppose that $\left(^{*}\right)$ is false. Then there exists some $\varepsilon>0$ such that for all $n \in \mathbb{N}$, there are $p_{n}, q_{n} \in \mathbb{N}$ with $p_{n}>q_{n} \geq n$ satisfying:

(i) $\varphi\left(d\left(x_{p_{n}}, x_{q_{n}}\right)\right) \geq \varepsilon$, and

(ii) $p_{n}$ is the smallest number greater than $q_{n}$ such that the condition $(i)$ holds.

Since

$$
\begin{aligned}
\varepsilon & \leq \varphi\left(d\left(x_{p_{n}}, x_{q_{n}}\right)\right) \\
& \leq \varphi\left(d\left(x_{p_{n}}, x_{p_{n}-1}\right)+d\left(x_{p_{n}-1}, x_{q_{n}}\right)\right) \\
& \leq \varphi\left(d\left(x_{p_{n}}, x_{p_{n}-1}\right)\right)+\varphi\left(d\left(x_{p_{n}-1}, x_{q_{n}}\right)\right) \\
& \leq \varphi\left(d\left(x_{p_{n}}, x_{p_{n}-1}\right)\right)+\varepsilon
\end{aligned}
$$

hence we conclude $\lim _{p \rightarrow \infty} \varphi\left(d\left(x_{p_{n}}, x_{q_{n}}\right)\right)=\varepsilon$. Since $\phi$ is subadditive and nondecreasing, we conclude

$$
\begin{aligned}
\varphi\left(d\left(x_{p_{n}}, x_{q_{n}}\right)\right) & \leq \varphi\left(d\left(x_{p_{n}}, x_{q_{n}+1}\right)+d\left(x_{p_{n}+1}, x_{q_{n}}\right)\right) \\
& \leq \varphi\left(d\left(x_{p_{n}}, x_{q_{n}+1}\right)\right)+\varphi\left(d\left(x_{p_{n}+1}, x_{q_{n}}\right)\right),
\end{aligned}
$$

and so

$$
\begin{aligned}
\varphi\left(d\left(x_{p_{n}}, x_{q_{n}}\right)\right)-\varphi\left(d\left(x_{p_{n}}, x_{p_{n}+1}\right)\right) & \leq \varphi\left(d\left(x_{p_{n}+1}, x_{q_{n}}\right)\right) \\
& \leq \varphi\left(d\left(x_{p_{n}}, x_{p_{n}+1}\right)+d\left(x_{p_{n}}, x_{q_{n}}\right)\right) \\
& \leq \varphi\left(d\left(x_{p_{n}}, x_{p_{n}+1}\right)\right)+\varphi\left(d\left(x_{p_{n}}, x_{q_{n}}\right)\right) .
\end{aligned}
$$

Letting $n \rightarrow \infty$, we also have

$$
\lim _{n \rightarrow \infty} \varphi\left(d\left(x_{p_{n}+1}, x_{q_{n}}\right)\right)=\varepsilon .
$$

Thus, there exists $i, 0 \leq i \leq m-1$ such that $p_{n}-q_{n}+i=1 \bmod m$ for infinitely many $n$. If $i=0$, then we have that for such $n$,

$$
\begin{aligned}
\varepsilon & \leq \varphi\left(d\left(x_{p_{n}}, x_{q_{n}}\right)\right) \\
& \leq \varphi\left(d\left(x_{p_{n}}, x_{p_{n}+1}\right)+d\left(x_{p_{n}+1}, x_{q_{n}+1}\right)+d\left(x_{q_{n}+1}, x_{q_{n}}\right)\right) \\
& \leq \varphi\left(d\left(x_{p_{n}}, x_{p_{n}+1}\right)\right)+\varphi\left(d\left(x_{p_{n}+1}, x_{q_{n}+1}\right)\right)+\varphi\left(d\left(x_{q_{n}+1}, x_{q_{n}}\right)\right) \\
& =\varphi\left(d\left(x_{p_{n}}, x_{p_{n}+1}\right)\right)+\varphi\left(d\left(f x_{p_{n}}, f x_{q_{n}}\right)\right)+\varphi\left(d\left(x_{q_{n}+1}, x_{q_{n}}\right)\right) \\
& \leq \varphi\left(d\left(x_{p_{n}}, x_{p_{n}+1}\right)\right)+\phi\left(\varphi\left(d\left(x_{p_{n}}, x_{q_{n}}\right)\right)\right)+\varphi\left(d\left(x_{q_{n}+1}, x_{q_{n}}\right)\right) .
\end{aligned}
$$

Letting $n \rightarrow \infty$. Then by, we have

$$
\varepsilon \leq 0+\lim _{n \rightarrow \infty} \phi\left(\varphi\left(d\left(x_{p_{n}}, x_{q_{n}}\right)\right)\right)+0<\varepsilon,
$$

a contradiction. Therefore $\lim _{n \rightarrow \infty} \varphi\left(d\left(x_{p_{n}}, x_{q_{n}}\right)\right)=0$, by the condition $\left(\phi_{3}\right)$, we also have $\lim _{n \rightarrow \infty} d\left(x_{p_{n}}, x_{q_{n}}\right)=0$. The case $i \neq 0$ is similar. Thus, $\left\{x_{n}\right\}$ is a Cauchy sequence. Since $X$ is complete, there exists $v \in \cup_{i=1}^{m} A_{i}$ such that $\lim _{n \rightarrow \infty} x_{n}=v$. Now for all $i=0,1$, $2, \ldots, m-1,\left\{f x_{m n-i}\right\}$ is a sequence in $A_{i}$ and also all converge to $v$. Since $A_{i}$ is clsoed for all $i=1,2, \ldots, m$, we conclude $v \in \cup_{i=1}^{m} A_{i}$, and also we conclude that $\cap_{i=1}^{m} A_{i} \neq \phi$. Since

$$
\begin{aligned}
\varphi(d(v, f v)) & =\lim _{n \rightarrow \infty} \varphi\left(d\left(f x_{m n}, f v\right)\right) \\
& \leq \lim _{n \rightarrow \infty} \phi\left(\varphi\left(d\left(f x_{m n-1}, v\right)\right)\right)=0,
\end{aligned}
$$


hence $\phi(d(v, f v))=0$, that is, $d(v, f v)=0, v$ is a fixed point of $f$.

Finally, to prove the uniqueness of the fixed point, let $\mu$ be another fixed point of $f$. By the cyclic character of $f$, we have $\mu, v \in \cap_{i=1}^{n} A_{i}$. Since $f$ is a cyclic weaker $\phi \circ \phi$-contraction, we have

$$
\begin{aligned}
\varphi(d(v, \mu)) & =\varphi(d(v, f \mu))=\lim _{n \rightarrow \infty} \varphi\left(d\left(f x_{m n}, f \mu\right)\right) \\
& \leq \lim _{n \rightarrow \infty} \phi\left(\varphi\left(d\left(f x_{m n-1}, \mu\right)\right)\right) \\
& <\varphi(d(v, \mu)),
\end{aligned}
$$

and this is a contradiction unless $\phi(d(v, \mu))=0$, that is, $\mu=v$. Thus $v$ is a unique fixed point of $f$.

Example 1 Let $X=\mathbb{R}^{3}$ and we define $d: X \times X \rightarrow[0, \infty)$ byd $(x, y)=\left|x_{1}-y_{1}\right|+\left|x_{2}-y_{2}\right|+\mid$ $x_{3}-y_{3} \mid$, for $x=\left(x_{1}, x_{2}, x_{3}\right), y=\left(y_{1}, y_{2}, y_{3}\right) \in X$, and let $A=\{(x, 0,0): x \in \mathbb{R}\}, B=\{(0, y, 0): y$ $\in \mathbb{R}\}, C=\{(0,0, z): z \in \mathbb{R}\}$ be three subsets of $X$. Define $f: A \cup B \cup C \rightarrow A \cup B \cup C$ by

$$
\begin{aligned}
& f((x, 0,0))=\left(0, \frac{1}{4} x, 0\right) ; \quad \text { for all } x \in \mathbb{R} ; \\
& f((0, y, 0))=\left(0,0, \frac{1}{4} y\right) ; \quad \text { for all } y \in \mathbb{R} ; \\
& f((0,0, z))=\left(\frac{1}{4} z, 0,0\right) ; \quad \text { for all } z \in \mathbb{R} .
\end{aligned}
$$

We define $\phi:[0, \infty) \rightarrow[0, \infty)$ by

$$
\phi(t)=\frac{1}{3} t \text { for } t \in[0, \infty),
$$

and $\phi:[0, \infty) \rightarrow[0, \infty)$ by

$$
\varphi(t)=\frac{1}{2} t \text { for } t \in[0, \infty) .
$$

Then $f$ is a cyclic weaker $\phi \circ \phi$-contraction and $(0,0,0)$ is the unique fixed point.

\section{Fixed point theory for the cyclic weaker $(\phi, \phi$-contractions}

The main purpose of this section is to present a generalization of Theorem 2 . In the section, we let $\phi:[0, \infty) \rightarrow[0, \infty)$ be a weaker Meir-Keeler function satisfying the following conditions:

$\left(\phi_{1}\right) \phi(t)>0$ for $t>0$ and $\phi(0)=0$;

$\left(\phi_{2}\right)$ for all $t \in(0, \infty),\left\{\phi^{n}(t)\right\}_{n \in \mathbb{N}}$ is decreasing;

$\left(\phi_{3}\right)$ for $t_{n} \in[0, \infty)$, if $\lim _{n \rightarrow \infty} t_{n}=\gamma$, then $\lim _{n \rightarrow \infty} \phi\left(t_{n}\right) \leq \gamma$.

And, let $\phi:[0, \infty) \rightarrow[0, \infty)$ be a non-decreasing and continuous function satisfying $\phi(t)>0$ for $t>0$ and $\phi(0)=0$.

We now state the notion of cyclic weaker $(\phi, \phi)$-contraction, as follows:

Definition 5 Let $(X, d)$ be a metric space, $m \in \mathbb{N}, A_{1}, A_{2}, \ldots, A_{m}$ nonempty subsets of $X$ and $X=\cup_{i=1}^{m} A_{i}$. An operator $f: X \rightarrow X$ is called a cyclic weaker $(\phi, \phi)$-contraction if

(i) $X=\cup_{i=1}^{m} A_{i}$ is a cyclic representation of $X$ with respect to $f$; 
(ii) for any $x \in A_{i}, y \in A_{i+1}, i=1,2, \ldots, m$,

$$
d(f x, f y) \leq \phi(d(x, y))-\varphi(d(x, y)),
$$

where $A_{m+1}=A_{1}$.

Theorem 4 Let $(X, d)$ be a complete metric space, $m \in \mathbb{N}, A_{1}, A_{2}, \ldots, A_{m}$ nonempty subsets of $X$ and $X=\cup_{i=1}^{m} A_{i}$. Let $f: X \rightarrow X$ be a cyclic weaker $(\phi, \phi)$-contraction. Then $f$ has a unique fixed point $z \in \cap_{i=1}^{m} A_{i}$.

Proof. Given $x_{0}$ and let $x_{n+1}=f x_{n}=f^{n+1} x_{0}$, for $n \in \mathbb{N} \cup\{0\}$. If there exists $n \in \mathbb{N} \cup\{0\}$ such that $x_{n_{0}+1}=x_{n_{0}}$, then we finished the proof. Suppose that $x_{n+1} \neq x_{n}$ for any $n \in \mathbb{N}$ $\cup\{0\}$. Notice that, for any $n>0$, there exists $i_{n} \in\{1,2, \ldots, m\}$ such that $x_{n-1} \in A_{i_{n}}$ and $x_{n} \in A_{i_{n}+1}$. Since $f: X \rightarrow X$ is a cyclic weaker $(\phi, \phi)$-contraction, we have that $n \in \mathbb{N}$

$$
\begin{aligned}
d\left(x_{n}, x_{n+1}\right) & =d\left(f x_{n-1}, f x_{n}\right) \\
& \leq \phi\left(d\left(x_{n-1}, x_{n}\right)\right)-\varphi\left(d\left(x_{n-1}, x_{n}\right)\right) \\
& \leq \phi\left(d\left(x_{n-1}, x_{n}\right)\right)
\end{aligned}
$$

and so

$$
\begin{aligned}
d\left(x_{n}, x_{n+1}\right) & \leq \phi\left(d\left(x_{n-1}, x_{n}\right)\right) \\
& \leq \phi\left(\phi\left(d\left(x_{n-2}, x_{n-1}\right)\right)=\phi^{2}\left(d\left(x_{n-2}, x_{n-1}\right)\right)\right. \\
& \leq \ldots \ldots \\
& \leq \phi^{n}\left(d\left(x_{0}, x_{1}\right)\right)
\end{aligned}
$$

Since $\left\{\phi^{n}\left(d\left(x_{0}, x_{1}\right)\right)\right\}_{n \in \mathbb{N}}$ is decreasing, it must converge to some $\eta \geq 0$. We claim that $\eta=$ 0 . On the contrary, assume that $\eta>0$. Then by the definition of weaker Meir-Keeler function $\phi$, there exists $\delta>0$ such that for $x_{0}, x_{1} \in X$ with $\eta \leq d\left(x_{0}, x_{1}\right)<\delta+\eta$, there exists $n_{0}$ $\in \mathbb{N}$ such that $\phi^{n_{0}}\left(d\left(x_{0}, x_{1}\right)\right)<\eta$. Since $\lim _{n \rightarrow \infty}, \phi^{n}\left(d\left(x_{0}, x_{1}\right)\right)=\eta$, there exists $p_{0} \in \mathbb{N}$ such that $\eta \leq \phi^{p}\left(d\left(x_{0}, x_{1}\right)\right)<\delta+\eta$, for all $p \geq p_{0}$. Thus, we conclude that $\phi^{p_{0}+n_{0}}\left(d\left(x_{0}, x_{1}\right)\right)<\eta$. So we get a contradiction. Therefore $\lim _{n \rightarrow \infty} \phi^{n}\left(d\left(x_{0}, x_{1}\right)\right)=0$, that is,

$$
\lim _{n \rightarrow \infty} d\left(x_{n}, x_{n+1}\right)=0 \text {. }
$$

Next, we claim that $\left\{x_{n}\right\}$ is a Cauchy sequence. We claim that the following result holds:

Claim: For every $\varepsilon>0$, there exists $n \in \mathbb{N}$ such that if $p, q \geq n$ with $p-q=1 \bmod m$, then $d\left(x_{p}, x_{q}\right)<\varepsilon$.

Suppose the above statement is false. Then there exists $\epsilon>0$ such that for any $n \in \mathbb{N}$, there are $p_{n}, q_{n} \in \mathbb{N}$ with $p_{n}>q_{n} \geq n$ with $p_{n}-q_{n}=1 \bmod m$ satisfying

$$
d\left(x_{q_{n}}, x_{p_{n}}\right) \geq \varepsilon .
$$

Now, we let $n>2 m$. Then corresponding to $q_{n} \geq n$ use, we can choose $p_{n}$ in such a way, that it is the smallest integer with $p_{n}>q_{n} \geq n$ satisfying $p_{n}-q_{n}=1 \bmod m$ and $d\left(x_{q_{n}}, x_{p_{n}}\right) \geq \varepsilon$. Therefore $d\left(x_{q_{n}}, x_{p_{n}-m}\right) \leq \varepsilon$ and

$$
\begin{aligned}
\varepsilon & \leq d\left(x_{q_{n}}, x_{p_{n}}\right) \\
& \leq d\left(x_{q_{n}}, x_{p_{n}-m}\right)+\sum_{i=1}^{m} d\left(x_{p_{n-i}}, x_{p_{n-i+1}}\right) \\
& <\varepsilon+\sum_{i=1}^{m} d\left(x_{p_{n-i}}, x_{p_{n-i+1}}\right) .
\end{aligned}
$$


Letting $n \rightarrow \infty$, we obtain that

$$
\lim _{n \rightarrow \infty} d\left(x_{q_{n}}, x_{p_{n}}\right)=\varepsilon
$$

On the other hand, we can conclude that

$$
\begin{aligned}
& \varepsilon \leq d\left(x_{q_{n}}, x_{p_{n}}\right) \\
& \quad \leq d\left(x_{q_{n}}, x_{q_{n+1}}\right)+d\left(x_{q_{n+1}}, x_{p_{n+1}}\right)+d\left(x_{x_{p_{n+1}}, p_{n}}\right) \\
& \quad \leq d\left(x_{q_{n}}, x_{q_{n+1}}\right)+d\left(x_{q_{n+1}}, x_{q_{n}}\right)+d\left(x_{q_{n}}, x_{p_{n}}\right)+d\left(x_{p_{n}}, x_{p_{n+1}}\right)+d\left(x_{x_{p_{n+1}}, p n}\right) .
\end{aligned}
$$

Letting $n \rightarrow \infty$, we obtain that

$$
\lim _{n \rightarrow \infty} d\left(x_{q_{n+1}}, x_{p_{n+1}}\right)=\varepsilon
$$

Since $x_{q_{n}}$ and $x_{p_{n}}$ lie in different adjacently labeled sets $A_{i}$ and $A_{i+1}$ for certain $1 \leq i \leq$ $m$, by using the fact that $f$ is a cyclic weaker $(\phi, \phi)$-contraction, we have

$$
d\left(x_{q_{n+1}}, x_{p_{n+1}}\right)=d\left(f x_{q_{n}}, f x_{p_{n}}\right) \leq \phi\left(d\left(x_{q_{n}}, x_{p_{n}}\right)\right)-\varphi\left(d\left(x_{q_{n}}, x_{p_{n}}\right)\right)
$$

Letting $n \rightarrow \infty$, by using the condition $\phi_{3}$ of the function $\phi$, we obtain that

$$
\varepsilon \leq \varepsilon-\varphi(\varepsilon)
$$

and consequently, $\phi(\epsilon)=0$. By the definition of the function $\phi$, we get $\epsilon=0$ which is contraction. Therefore, our claim is proved.

In the sequel, we shall show that $\left\{x_{n}\right\}$ is a Cauchy sequence. Let $\varepsilon>0$ be given. By our claim, there exists $n_{1} \in \mathbb{N}$ such that if $p, q \geq n_{1}$ with $p-q=1 \bmod m$, then

$$
d\left(x_{p}, x_{q}\right) \leq \frac{\varepsilon}{2}
$$

Since $\lim _{n \rightarrow \infty} d\left(x_{n}, x_{n+1}\right)=0$, there exists $n_{2} \in \mathbb{N}$ such that

$$
d\left(x_{n}, x_{n+1}\right) \leq \frac{\varepsilon}{2 m}
$$

for any $n \geq n_{2}$.

Let $p, q \geq \max \left\{n_{1}, n_{2}\right\}$ and $p>q$. Then there exists $k \in\{1,2, \ldots, m\}$ such that $p-q=$ $k \bmod m$. Therefore, $p-q+j=1 \bmod m$ for $j=m-k+1$, and so we have

$$
\begin{aligned}
d\left(x_{q}, x_{p}\right) & \leq d\left(x_{q}, x_{p+j}\right)+d\left(x_{p+j}, x_{p+j-1}\right)+\cdots+d\left(x_{p+1}, x_{p}\right) \\
& \leq \frac{\varepsilon}{2}+j \times \frac{\varepsilon}{2 m} \\
& \leq \frac{\varepsilon}{2}+m \times \frac{\varepsilon}{2 m}=\varepsilon .
\end{aligned}
$$

Thus, $\left\{x_{n}\right\}$ is a Cauchy sequence. Since $X$ is complete, there exists $v \in \cup_{i=1}^{m} A_{i}$ such that $\lim _{n \rightarrow \infty} x_{n}=v$. Since $X=\cup_{i=1}^{m} A_{i}$ is a cyclic representation of $X$ with respect to $f$, the sequence $\left\{x_{n}\right\}$ has infinite terms in each $A_{i}$ for $i \in\{1,2, \ldots, m\}$. Now for all $i=1,2, \ldots$, $m$, we may take a subsequence $\left\{x_{n_{k}}\right\}$ of $\left\{x_{n}\right\}$ with $x_{n_{k}} \in A_{i-1}$ and also all converge to $v$. Since

$$
\begin{aligned}
d\left(x_{n_{k+1}}, f v\right) & =d\left(f x_{n_{k}}, f v\right) \\
& \leq \phi\left(d\left(x_{n_{k}}, v\right)\right)-\varphi\left(d\left(x_{n_{k}}, v\right)\right) \\
& \leq \phi\left(d\left(x_{n_{k}}, v\right)\right)
\end{aligned}
$$


Letting $k \rightarrow \infty$, we have

$$
d(v, f v) \leq 0,
$$

and so $v=f v$.

Finally, to prove the uniqueness of the fixed point, let $\mu$ be the another fixed point of $f$. By the cyclic character of $f$, we have $\mu, v \in \cap_{i=1}^{n} A_{i}$. Since $f$ is a cyclic weaker $(\phi$, $\phi)$-contraction, we have

$$
\begin{aligned}
d(v, \mu) & =d(v, f \mu) \\
& =\lim _{n \rightarrow \infty} d\left(x_{n_{k+1}}, f \mu\right) \\
& =\lim _{n \rightarrow \infty} d\left(f x_{n_{k}}, f \mu\right) \\
& \leq \lim _{n \rightarrow \infty}\left[\phi\left(d\left(x_{n_{k}}, \mu\right)\right)-\varphi\left(d\left(x_{n_{k}}, \mu\right)\right)\right] \\
& \leq d(\nu, \mu)-\varphi(d(v, \mu)),
\end{aligned}
$$

and we can conclude that

$$
\varphi(d(\nu, \mu))=0 .
$$

So we have $\mu=v$. We complete the proof.

Example 2 Let $X=[-1,1]$ with the usual metric. Suppose that $A_{1}=[-1,0]=A_{3}$ and $A_{2}=[0,1]=A_{4}$. Define $f: X \rightarrow X$ by $f(x)=\frac{-x}{6}$ for all $x \in X$, and let $\phi, \phi:[0, \infty) \rightarrow[0$, $\infty)$ be $\phi(t)=\frac{1}{2}, \varphi(t)=\frac{t}{4}$. Then $f$ is a cyclic weaker $(\phi, \phi)$-contraction and 0 is the unique fixed point.

Example 3 Let $X=\mathbb{R}^{+}$with the metric $d: X \times X \rightarrow \mathbb{R}^{+}$given by

$$
d(x, y)=\max \{x, y\}, \quad \text { for } \quad x, y \in X .
$$

Let $A_{1}=A_{2}=\ldots=A_{m}=\mathbb{R}^{+}$. Define $f: X \rightarrow X$ by

$$
f(x)=\frac{x^{2}}{77} \quad \text { for } \quad x \in X
$$

and let $\phi, \phi:[0, \infty) \rightarrow[0, \infty)$ be $\varphi(t)=\frac{t^{3}}{2(t+2)}$ and

$$
\phi(t)=\left\{\begin{array}{cl}
\frac{2 t^{3}}{3 t+8}, & \text { if } t \geq 1 \\
\frac{t^{2}}{2}, & \text { if } t<1
\end{array}\right.
$$

Then $f$ is a cyclic weaker $(\phi, \phi)$-contraction and 0 is the unique fixed point.

Example 4 Let $X=\mathbb{R}^{3}$ and we define $d: X \times X \rightarrow[0, \infty)$ by

$$
d(x, y)=\max \left\{\left|x_{1}-y_{1}\right|,\left|x_{2}-y_{2}\right|,\left|x_{3}-y_{3}\right|,\right\}
$$

for $x=\left(x_{1}, x_{2}, x_{3}\right), y=\left(y_{1}, y_{2}, y_{3}\right) \in X$, and let $A=\{(x, 0,0): x \in[0,1]\}, B=\{(0, y, 0): y \in$ $[0,1]\}, C=\{(0,0, z): z \in[0,1]\}$ be three subsets of $X$. 
Define $f: A \cup B \cup C \rightarrow A \cup B \cup C$ by

$$
\begin{aligned}
& f((x, 0,0))=\left(0, \frac{1}{8} x^{2}, 0\right) ; \quad \text { for all } x \in[0,1] ; \\
& f((0, y, 0))=\left(0,0, \frac{1}{8} y^{2}\right) ; \quad \text { for all } y \in[0,1] ; \\
& f((0,0, z))=\left(\frac{1}{8} z^{2}, 0,0\right) ; \quad \text { for all } z \in[0,1] .
\end{aligned}
$$

We define $\phi:[0, \infty) \rightarrow[0, \infty)$ by

$$
\phi(t)=\frac{t^{2}}{t+1} \text { for } t \in[0, \infty)
$$

and $\phi:[0, \infty) \rightarrow[0, \infty)$ by

$$
\varphi(t)=\frac{t^{2}}{t+2} \text { for } t \in[0, \infty) .
$$

Then $f$ is a cyclic weaker $(\phi, \phi)$-contraction and $(0,0,0)$ is the unique fixed point.

\section{Acknowledgements}

The authors would like to thank referee(s) for many useful comments and suggestions for the improvement of the article.

\section{Competing interests}

The authors declare that they have no competing interests.

\section{Received: 14 November 2011 Accepted: 16 February 2012 Published: 16 February 2012}

\section{References}

1. Banach, S: Sur les operations dans les ensembles abstraits et leur application aux equations integerales. Fund Math. 3, 133-181 (1922)

2. Boyd, DW, Wong, SW: On nonlinear contractions. Proc Am Math Soc. 20, 458-464 (1969). doi:10.1090/S0002-9939-19690239559-9

3. Aydi, H, Karapinar, E, Shatnawi, W: Coupled fixed point results for $(\psi-\varphi)$-weakly contractive condition in ordered partial metric spaces. Comput Math Appl. 62(12):4449-4460 (2011). doi:10.1016/j.camwa.2011.10.021

4. Karapinar, E: Weak $\varphi$-contraction on partial metric spaces and existence of fixed points in partially ordered sets. Mathematica Aeterna. 1(4):237-244 (2011)

5. Kirk, WA, Srinivasan, PS, Veeramani, P: Fixed points for mappings satisfying cyclical contractive conditions. Fixed Point Theory. 4(1):79-89 (2003)

6. Rus, IA: Cyclic representations and fixed points. Ann T Popoviciu, Seminar Funct Eq Approx Convexity. 3, 171-178 (2005)

7. Păcurar, M, Rus, IA: Fixed point theory for cyclic $\varphi$-contractions. Nonlinear Anal. 72(3-4):2683-2693 (2010)

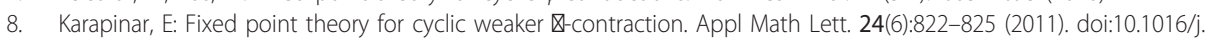
aml.2010.12.016

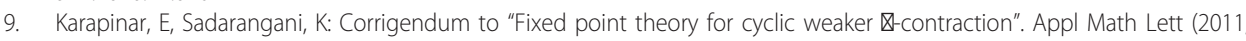
in press). [Appl. Math. Lett. Vol.24(6), 822-825.]

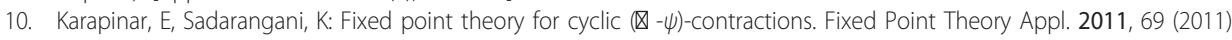
doi:10.1186/1687-1812-2011-69

11. Meir, A, Keeler, E: A theorem on contraction mappings. J Math Anal Appl. 28, 326-329 (1969). doi:10.1016/0022-247X (69)90031-6

doi:10.1186/1687-1812-2012-17

Cite this article as: Chen: Fixed point theory for the cyclic weaker Meir-Keeler function in complete metric spaces. Fixed Point Theory and Applications 2012 2012:17. 